\title{
The $\{\beta\}$-expansion formalism in perturbative $Q C D$ and its extension
}

\author{
A.L. Kataev ${ }^{a, b}$ and S.V. Mikhailov ${ }^{c}$ \\ ${ }^{a}$ Institute for Nuclear Research of the Academy of Sciences of Russia, \\ 60th October Anniversary Prospect 7a, 117312, Moscow, Russia \\ ${ }^{b}$ Moscow Institute of Physics and Technology, \\ Institutskii per. 9, 141700, Dolgoprudny, Moscow Region, Russia \\ ${ }^{c}$ Bogoliubov Laboratory of Theoretical Physics, JINR, \\ Joliot-Curie 6, 141980 Dubna, Russia \\ E-mail: kataev@ms2.inr.ac.ru, mikhs@theor.jinr.ru
}

ABSTRACT: We discuss the $\{\beta\}$-expansion for renormalization group invariant quantities tracing this expansion to the different contractions of the corresponding incomplete BPHZ $R$-operation. All of the coupling renormalizations, which follow from these contractions, should be taken into account for the $\{\beta\}$-expansion. We illustrate this feature considering the nonsinglet Adler function $D^{\mathrm{NS}}$ in the third order of perturbation. We propose a generalization of the $\{\beta\}$-expansion for the renormalization group covariant quantities - the $\{\beta, \gamma\}$-expansion.

KEYwords: Renormalization Group, Renormalization Regularization and Renormalons ARXIV EPRINT: 1607.08698 


\section{Contents}

1 Introduction 1

2 The Adler function and its $\{\boldsymbol{\beta}\}$-expansion 3

3 The Adler function calculations in QCD $\mathbf{5}$

3.1 The renormalization relations for the Adler function in QCD 5

3.2 The Adler function in terms of photon anomalous dimension $\gamma_{\mathrm{ph}}^{\mathrm{EM}} \quad 6$

4 The structure of the $R^{\prime}$-operation and the $\{\beta\}$-expansion 8

5 Generalized $\{\beta, \gamma\}$-expansion $\quad 11$

6 Conclusion 13

A Explicit formulas for the elements of $D$ and $\beta$-function coefficients $\quad 13$

\section{Introduction}

In the last few years, interest in the construction of the high-order generalizations of the Brodsky-Lepage-Mackenzie (BLM) approach [1] for fixing the scales in the perturbative QCD expansions of the renormalization-group invariant (RGI) quantities in powers of the coupling constant $\alpha_{s}\left(\mu^{2}\right)$ was renewed (see, e.g., $[2-5,8]$ ). A special feature of the new class of the BLM based procedures is the absorption into new scales of those terms that are proportional to the coefficients of the QCD $\beta$-function fill factors. These new perturbative expansions are based on the expansion of the terms of the massless perturbative QCD series for the RGI-invariant quantities in the powers and the products of powers of the coefficients of the QCD $\beta$-function. It is called the $\{\beta\}$-expansion formalism (see, e.g., [5]) and was first proposed in [2] as the double series representation for the quantity in the parameters $\alpha_{s}^{n}$ and $\beta_{i}^{l}$ - the powers of the $\beta$-function coefficients ("matrix representation"). Then this $\{\beta\}$-expansion was intensively studied in [5-9] in the case of QCD supplemented with multiplets of gluions, which are the elements of general SUSY extension of QCD theory. In $[10-12]$, the variants of the $\{\beta\}$-expansion were used in QCD without additional degrees of freedom, while in [13] the variants of the $\{\beta\}$-expansion procedure were applied in both the cases, namely in QCD with and without additional gluino degrees of freedom. Note that in general the $\{\beta\}$-expansion is formulated for the RGI quantities, initially evaluated within the class of minimal subtraction (MS)-schemes (see, e.g., $[2,11]$ ).

At the next step of elaboration of the modern generalizations of the BLM approach all the terms, proportional to the coefficients of the QCD $\beta$-function, are transferred into the scales $\mu^{2}$ of the powers of $\alpha_{s}\left(\mu^{2}\right)$ (or $a_{s}\left(\mu^{2}\right)=\alpha_{s}\left(\mu^{2}\right) /(4 \pi)$ ) in the corresponding series (see, 
e.g., $[2,10-13])$. However, there are still several points of view how to use the BLM approach that is generalized in this way and how to fix the concrete content of the $\{\beta\}$-dependent elements in the perturbative coefficients in the framework of the MS-like renormalization scheme. They appear during the development and study of the applications of the BLM generalizations in practice. They are pushed forward mainly by two groups: the authors of the present work and the authors of the "Principle of Maximum Conformality" (PMC) also based on the $\{\beta\}$-expansion [10-12]. The first theoretical disagreement finally leads to different values of the $\beta$-dependent elements of the expansion coefficients of the RGI quantities $^{1}$ to different results for new BLM-type scales, and to different points of view on the scheme-dependence of the $\{\beta\}$-independent terms in the certain PMC-type series. The second disagreement is related to different interpretations of the $\{\beta\}$-expansion for two related representations of the $e^{+} e^{-}$-annihilation Adler function $D^{\mathrm{EM}}\left(a_{s}\right)$ which, contrary to the considerations presented in $[3,12,13]$, should lead to the identical results, in full agreement with the basis of the incomplete BPHZ R-operation described in detail in [14].

The first part of this work is devoted to the proof of the latter statement. In section 2 we discuss the definitions and properties of the photon vacuum polarization function $\Pi^{\mathrm{EM}}\left(a_{s}\right)$ and of its anomalous dimension $\gamma_{p h}^{E M}\left(a_{s}\right)$ following the detailed considerations in $[15,16]$. In section 3 we recall to readers the details of the calculations of $D^{\mathrm{EM}}$. The aim is to demonstrate how the definition of the $\{\beta\}$-expansion proposed in [2] can be realized for the case of the $O\left(a_{s}^{4}\right)$ representation of the $D^{\mathrm{EM}}$-function explicitly presented in [17] in terms of the photon anomalous dimension and the polarization function $\Pi^{\mathrm{EM}}\left(a_{s}\right)$, which was used in the consideration of [12]. We shall clarify the statement already made in [5] that the careful application of the $\{\beta\}$-expansion in this case leads to the same results for the $\{\beta\}$-expansion obtained in [2] without involving into consideration this presentation for the $D^{\mathrm{EM}}$-function with the photon anomalous dimension as well. This clarification should be compared with the non-completed analysis presented in $[3,12,13]$.

In section 4 , we demonstrate that the $\{\beta\}$-expansion of the RGI-invariant quantities (in the MS-like schemes) can be traced to the $R^{\prime}$-operation, i.e. the incomplete BPHZ R-operation. We recall, following the studies in $[15,16]$, that the subtraction of all QCD subdivergences from the bare photon vacuum polarization function, which enter into the definition of the $D^{\mathrm{EM}}$-function, is equivalent to the renormalization of the bare QCD coupling constant $a_{s B}$ in this expression. This allows us to show in a more formal way why the application of the $\{\beta\}$-expansion approach to the different presentations of the Adler function through $\gamma_{p h}^{E M}\left(a_{s}\right)$ and $\Pi^{\mathrm{EM}}\left(a_{s}\right)$ and through the Källén-Lehmann dispersion representation give identical results.

In section 5 , we generalize the $\{\beta\}$-expansion to the case of the RG-covariant quantities, which have their own anomalous dimensions. In this case, the structure of the expansion of the coefficient will differ from the structure of the $\{\beta\}$-expansion of the RG-invariant quantity - it will contain new terms, which are proportional to the coefficients of the corresponding anomalous dimension. The combined consideration of these terms forms the

\footnotetext{
${ }^{1}$ In the recent paper the general form of the $\{\beta\}$-expended expressions for these coefficients is the same as the one, introduced in [2] and used in [5, 6], but the concrete coefficients remain different.
} 
basis for the new $\{\beta, \gamma\}$-expansion. We emphasize the necessity of further development of the optimization of the related series in the spirit of the generalized BLM approach.

\section{The Adler function and its $\{\boldsymbol{\beta}\}$-expansion}

Let us start first with the expression for the vacuum polarization tensor, which is related to the polarization function $\Pi^{\mathrm{EM}}$ as

$$
\Pi_{\mu \nu}^{\mathrm{EM}}\left(q, a_{s}\right)=i \int e^{i q x}\left\langle 0\left|J_{\mu}^{\mathrm{EM}}(x) J_{\nu}^{\mathrm{EM}}(0)\right| 0\right\rangle d^{d} x=\left(q_{\mu} q_{\nu}-g_{\mu \nu} q^{2}\right) \Pi^{\mathrm{EM}}\left(L, a_{s}\right) .
$$

Here $J_{\mu}^{\mathrm{EM}}(x)=\sum_{i} q_{i} \cdot \bar{\psi}_{i} \gamma_{\mu} \psi_{i}, q_{i}$ are the electromagnetic charges of quarks, $-q^{2}=Q^{2}$, $L=\ln \left(\mu^{2} / Q^{2}\right)$. Due to the vector current conservation the r.h.s. of eq. (2.1) is transverse. The vacuum polarization function $\Pi^{\mathrm{EM}}\left(L, a_{s}\right)$ contains the non-singlet (NS) and singlet (SI) contributions

$$
\Pi^{\mathrm{EM}}\left(L, a_{s}\right)=\left(\sum_{i} q_{i}^{2}\right) \Pi^{\mathrm{NS}}\left(L, a_{s}\right)+\left(\sum_{i} q_{i}\right)^{2} \Pi^{\mathrm{SI}}\left(L, a_{s}\right) .
$$

The latter ones appear for the first time at the four-loop level [18-20]. The Adler function $D^{\mathrm{EM}}$ is a widely used characteristic of the $e^{+} e^{-}$-annihilation to a hadrons process, namely

$$
D^{\mathrm{EM}}\left(L, a_{s}\right)=-12 \pi^{2} Q^{2} \frac{d}{d Q^{2}} \Pi^{\mathrm{EM}}\left(L, a_{s}\right)=Q^{2} \int_{4 m_{\pi}^{2}}^{\infty} \frac{R(s)}{\left(s+Q^{2}\right)^{2}} d s,
$$

which was introduced in [21] in the Euclidean region. In the r.h.s. of this Källén-Lehmann representation the spectral function $R(s)=-12 \pi \operatorname{Im} \Pi^{\mathrm{EM}}(-\mathrm{s}+\mathrm{i} \epsilon)$ is related to the QCD expression for the total cross-section of the $e^{+} e^{-}$-annihilation to a hadrons process as $R(s)=\sigma_{\text {tot }}\left(e^{+} e^{-} \rightarrow\right.$ hadrons $) / \sigma\left(e^{+} e^{-} \rightarrow \mu^{+} \mu^{-}\right)$, where $\sigma\left(e^{+} e^{-} \rightarrow \mu^{+} \mu^{-}\right)=4 \pi \alpha_{e}^{2} /(3 s)$ is the theoretical normalization factor.

Following eq. (2.2) one can decompose the Adler function to the NS and SI parts as

$$
D^{\mathrm{EM}}\left(L, a_{s}\right)=D^{\mathrm{NS}}\left(L, a_{s}\right)+D^{\mathrm{SI}}\left(L, a_{s}\right) .
$$

This function is the renormalization-group (RG) invariant quantity and therefore it obeys the standard RG equation without anomalous dimension, namely

$$
\left(\frac{\partial}{\partial L}+\beta\left(a_{s}\right) \frac{\partial}{\partial a_{s}}\right) D^{\mathrm{EM}}\left(L, a_{s}\right)=0
$$

where the QCD $\beta$-function is

$$
\mu^{2} \frac{\partial a_{s}\left(\mu^{2}\right)}{\partial \mu^{2}}=\beta\left(a_{s}\right)=-a_{s}^{2} \sum_{i \geq 0} \beta_{i} a_{s}^{i} .
$$

In the normalization conditions we used $a_{s}=\alpha_{s} /(4 \pi)$; the first coefficient of this function reads $\beta_{0}=(11 / 3) C_{\mathrm{A}}-(4 / 3) T_{\mathrm{R}} n_{f}$. The solution of eq. (2.5) reads:

$$
D^{\mathrm{EM}}\left(L ; a_{s}\left(\mu^{2}\right)\right)=D^{\mathrm{EM}}\left(0 ; a_{s}\left(Q^{2}\right)\right) .
$$


We shall consider here the NS contribution to eq. (2.4), which we write down $[2,5]$ as

$$
D^{\mathrm{NS}}\left(a_{s}\left(Q^{2}\right)\right)=1+\sum_{i \geq 1} d_{l}^{\mathrm{NS}} a_{s}^{l}\left(Q^{2}\right)=1+d_{1}^{\mathrm{NS}} \cdot \sum_{l \geq 1} d_{l} a_{s}^{l}\left(Q^{2}\right)
$$

where the coefficients $d_{l}^{\mathrm{NS}}$ are considered within the class of the MS-like ultraviolet (UV) schemes. The coefficients $d_{l}=d_{l}^{\mathrm{NS}} / d_{1}^{\mathrm{NS}}$, the overall normalization factor $d_{1}^{\mathrm{NS}}=3 \mathrm{C}_{\mathrm{F}}$ that is more appropriate for the BLM like optimization. The $\beta$-expansion representation introduced in [2] prescribes to decompose these coefficients in the last equation of eq. (2.8) in the following way (see [6] as well):

$$
\begin{aligned}
d_{1} & =d_{1}[0]=1 \\
d_{2} & =\beta_{0} d_{2}[1]+d_{2}[0] \\
d_{3} & =\beta_{0}^{2} d_{3}[2]+\beta_{1} d_{3}[0,1]+\beta_{0} d_{3}[1]+d_{3}[0], \\
d_{4} & =\beta_{0}^{3} d_{4}[3]+\beta_{1} \beta_{0} d_{4}[1,1]+\beta_{2} d_{4}[0,0,1]+\beta_{0}^{2} d_{4}[2]+\beta_{1} d_{4}[0,1]+\beta_{0} d_{4}[1] \\
& \quad+d_{4}[0], \\
& \vdots \\
d_{N} & =\beta_{0}^{N-1} d_{N}[N-1]+\cdots+d_{N}[0]
\end{aligned}
$$

where $\beta_{i}$ are the coefficients of the $\beta$-function in eq. (2.6); the notation $i_{0}, i_{1}, \ldots$ for the arguments of $d_{n}\left[i_{0}, i_{1}, \ldots\right]$ denotes the powers of $\beta_{0}, \beta_{1}, \ldots$. The decompositions in eqs. (2.9) should contain all information about strong charge renormalization by means of using there all the possible $\beta_{i}$-terms. For the reader's convenience we present the explicit forms of the decomposition in $(2.9 \mathrm{~b}),(2.9 \mathrm{c})$ in appendix A for the case of QCD supplemented with multiplets of MSSM gluinos.

As follows from the studied in $[15,16]$ renormalization prescriptions, which will be presented in details in section 3, the expression for the Adler $D$-function in eq. (2.4) can also be written in the following form [17]:

$$
D^{\mathrm{EM}}\left(L, a_{s}\right)=12 \pi^{2}\left(\gamma_{\mathrm{ph}}^{\mathrm{EM}}\left(a_{s}\right)-\beta\left(a_{s}\right) \frac{d}{d a_{s}} \Pi^{\mathrm{EM}}\left(L, a_{s}\right)\right)
$$

where $\gamma_{\mathrm{ph}}^{\mathrm{EM}}\left(a_{s}\right)$ is the QCD anomalous dimension of the photon vacuum polarization, which is defined as

$$
\gamma_{\mathrm{ph}}^{\mathrm{EM}}\left(a_{s}\right)=\left.\mu^{2} \frac{d}{d \mu^{2}} \Pi^{\mathrm{EM}}\left(L, a_{s}\right)\right|_{L=0}
$$

In the discussions below we shall clarify that the coefficients of the photon anomalous dimension $\gamma_{\mathrm{ph}}^{\mathrm{EM}}\left(a_{s}\right)$ should not be neglected in the process of construction of the $\{\beta\}$ expansion formalism of [2] and of the careful construction of the PMC scale-setting prescription for the $D$-function (for the consideration of this topic at the next-to-next-to-leading order (NNLO) see [5] as well). 


\section{The Adler function calculations in QCD}

\subsection{The renormalization relations for the Adler function in QCD}

In our further discussions we shall use the MS-like scheme renormalization prescriptions for the Adler $D$-function, which were described in detail in $[15,16]$. They were used in the process of evaluation of the 2 nd order perturbative QCD correction to the $D$-function and to its spectral density $R(s)$ (in brief the result was published in [22]). The same renormalization prescriptions were used for the calculations of the 3rd and 4th order QCD corrections to $\Pi^{\mathrm{EM}}$ and $D^{\mathrm{EM}}$ in $[17,18]$. Following these prescriptions one should consider first the renormalization equation for the inverse photon propagator in $\mathrm{QCD}$

$$
1+a \Pi^{\mathrm{EM}}\left(L, a_{s}\right)=Z_{\mathrm{ph}}\left(1+a_{B} \Pi_{B}^{\mathrm{EM}}\left(L, a_{s B}\right)\right) .
$$

Here $Z_{\mathrm{ph}}$ is the photon renormalization constant, $\Pi^{\mathrm{EM}}\left(L, a_{s}\right)$ and $\Pi_{B}^{\mathrm{EM}}\left(L,, a_{s B}\right)$ are the renormalized and unrenormalized (bare) photon polarization functions, respectively. Due to the Ward identity, the bare electromagnetic coupling $a_{B}$ is related to the renormalized one $a$ as $Z_{\mathrm{ph}} a_{B}=a=\alpha_{e} /(4 \pi)$, while the QCD bare coupling $a_{s B}$ is related to the renormalized one as $Z_{a_{s}}^{-1} a_{s B}=a_{s}=\alpha_{s} /(4 \pi)$. In the class of MS-like schemes $Z_{\mathrm{ph}}$ has the following form:

$$
Z_{\mathrm{ph}}=1+a \cdot Z=1+a \cdot \sum_{l=1} a_{s}^{l-1} \sum_{k=1}^{l} Z_{l,-k} \varepsilon^{-k},
$$

where $\varepsilon=(4-d) / 2$ is the parameter of the dimensional regularization, $a_{s}-$ renormalized strong coupling. The QCD expression for the bare photon polarization function $\Pi_{B}^{\mathrm{EM}}\left(L, a_{s B}\right)$ in eq. (3.1) reads

$$
\Pi_{B}^{\mathrm{EM}}\left(L, a_{s B}\right)=\sum_{j=1}^{\infty} a_{s B}^{j-1} \exp (L j \varepsilon) \sum_{i=-j}^{\infty} \Pi_{j, i} \varepsilon^{i},
$$

where $\Pi_{j, i}$ are the elements of expansion of $\Pi_{B}^{\mathrm{EM}}$ in the double series. To get the renormalized expression for $\Pi^{\mathrm{EM}}\left(L, a_{s}\right)$, which determines eventually the renormalized Adler function, one would use $Z_{\mathrm{ph}} a_{B}=a$ (the Ward identity) for electromagnetic coupling and rewrite eq. (3.1) in terms of $Z=\left(Z_{\mathrm{ph}}-1\right) / a$ :

$$
\Pi^{\mathrm{EM}}\left(L, a_{s}\right)=Z+\Pi_{B}^{\mathrm{EM}}\left(L, a_{s B}\right) .
$$

We take into account the known relation between the bare coupling $a_{s B}$ and renormalized one $a_{s}$ at the $O\left(a_{s}^{3}\right)$ level,

$$
a_{s B}=a_{s}+a_{s}^{2}\left(-\beta_{0} / \varepsilon\right)+a_{s}^{3}\left(\beta_{0}^{2} / \varepsilon^{2}-\beta_{1} / \varepsilon\right)+O\left(a_{s}^{4}\right),
$$

and then substitute the expression for $Z$ in eq. (3.2) and eq. (3.3) together with eq. (3.5) into the r.h.s. of eq. (3.4) to obtain various relations between the pole parts $Z_{l,-k}$ and the 
elements $\Pi_{j, i}$. Using these relations in the definition of eq. $(2.3)$ for $D^{\mathrm{EM}}$ leads $[15,16]$ to the following expressions for the first perturbation coefficients $d_{i}^{N S}$

$$
\begin{aligned}
& d_{1}^{\mathrm{NS}}=-2 Z_{2,-1}, \\
& d_{2}^{\mathrm{NS}}=-3 Z_{3,-1}+\beta_{0} \Pi_{2,0} .
\end{aligned}
$$

Further application of the same renormalization prescriptions gives

$$
d_{3}^{\mathrm{NS}}=-4 Z_{4,-1}+2 \beta_{0} \Pi_{3,0}+\beta_{1} \Pi_{2,0}+2 \beta_{0}^{2} \Pi_{2,1} .
$$

The latter was obtained in [18]. As we have already discussed after eq. (3.5), the r.h.s. of eqs. (3.6a) $-(3.6 \mathrm{c})$ can be rewritten also in terms $\Pi_{j, i}$ instead of $Z_{l,-1}$, namely

$$
\begin{aligned}
& d_{1}^{\mathrm{NS}}=2 \Pi_{2,-1}, \\
& d_{2}^{\mathrm{NS}}=3 \Pi_{3,-1}-2 \beta_{0} \Pi_{2,0}, \\
& d_{3}^{\mathrm{NS}}=4 \Pi_{4,-1}-6 \beta_{0} \Pi_{3,0}-\beta_{1} \Pi_{2,0}+6 \beta_{0}^{2} \Pi_{2,1} .
\end{aligned}
$$

In the discussions below we will prove that eqs. (3.6a)-(3.6c), which contain the coefficients of the photon anomalous dimension function of eq. (2.11), i.e. the terms $-l Z_{l,-1}$, give the $\{\beta\}$-expanded structure for $d_{l-1}^{\text {NS }}$ coefficients, which is identical to the one formulated in [2] (see, e.g., eqs. (2.9a)-(2.9c) presented above).

\subsection{The Adler function in terms of photon anomalous dimension $\gamma_{\mathrm{ph}}^{\mathrm{EM}}$}

Here we consider the results for $d^{\mathrm{NS}}$ in eqs. (3.6a)-(3.6c) from the point of view of the general formula, eq. (2.10). We start with the expansion of the renormalized polarization function, which follows from the definition (2.2)

$$
\Pi^{\mathrm{EM}}\left(a_{s}\right)=\left.\Pi^{\mathrm{EM}}\left(L, a_{s}\right)\right|_{L=0} \equiv \frac{d_{R}}{(4 \pi)^{2}} \sum_{i \geq 1} a_{s}^{i-1} \Pi_{i}^{\mathrm{R}},
$$

where we supply the coefficients of this expansion with superscript $\mathrm{R}$ to distinguish them from the ones in expansion (3.3) of the bar $\Pi_{B}^{\mathrm{EM}}$. The corresponding anomalous dimension of the photon vacuum polarization function, already defined in eq. (2.11),

$$
\gamma_{\mathrm{ph}}^{\mathrm{EM}}\left(a_{s}\right)=\left.\mu^{2} \frac{d}{d \mu^{2}} \Pi^{\mathrm{EM}}\left(L, a_{s}\right)\right|_{L=0},
$$

can be derived in the MS-scheme from the first pole coefficient $Z_{-1}\left(a_{s}\right)$ of $Z$ in the expansion $(3.2)$

$$
\gamma_{\mathrm{ph}}^{\mathrm{EM}}\left(a_{s}\right)=-\partial_{a_{s}}\left[a_{s} \sum_{l \geq 1} a_{s}^{l-1} Z_{l,-1}\right]=\sum_{l \geq 1} a_{s}^{l-1}\left(-l Z_{l,-1}\right)=\frac{d_{R}}{(4 \pi)^{2}} \sum_{j \geq 0} a_{s}^{j} \gamma_{j} .
$$

Thus, the first terms in the r.h.s. of eqs. (3.6a)-(3.6c), namely $-2 Z_{2,-1},-3 Z_{3,-1},-4 Z_{4,-1}$, are the perturbation theory coefficients of $\gamma_{\mathrm{ph}}^{\mathrm{EM}}$ defined in the r.h.s. of eq. (3.9). Note that the sum $\sum_{l \geq 1} a_{s}^{l-1} Z_{l,-1}$ in the 1.h.s. of eq. (3.9) forms the coefficient $Z_{-1}\left(a_{s}\right)$. The 
analytical expressions for $\gamma_{i}$ and $\Pi_{j}^{\mathrm{R}}$, were calculated in the $\overline{\mathrm{MS}}$-scheme up to $i=4, j=4$ in [17]. Both terms $\gamma_{\mathrm{ph}}^{\mathrm{EM}}\left(a_{s}\right)$ and $\Pi^{\mathrm{EM}}\left(a_{s}\right)$ in the r.h.s. of eq. (2.10) contain the traces of $a_{s}$ renormalization accumulated in the $\beta$-function (more precisely in its coefficients). In contrast to the conclusion, presented in $[3,12,13]$, both these terms should be taken into account in the $\{\beta\}$-expansion. This expansion of $D^{\mathrm{EM}}$ in order of $a_{s}^{2}$ has already been discussed in section 3.3 of [5], where the values for $\Pi_{2}^{\mathrm{R}}$ and $\gamma_{3}$ from [17] were used (there, the numeration of $\Pi_{i}^{\mathrm{R}}$ in index $i$ is shifted by 1 less and coupling constant is $\left.a_{s}=\alpha_{s} / \pi\right)$. There was already shown that the $\beta_{0}$-part of $\gamma_{2}=-3 Z_{3,-1}$ (these contributions are underlined in the expression presented below) together with the second term $\beta_{0} \Pi_{2}^{\mathrm{R}}$ enters into the coefficient of $d_{2}^{N S}$ in eq. (3.7b) as

$$
\begin{aligned}
& d_{2}^{\mathrm{NS}}=\frac{3}{4} \gamma_{2}+\frac{3}{4} \beta_{0} \Pi_{2}^{\mathrm{R}}=3 \mathrm{C}_{\mathrm{F}} \cdot\left[\underline{\left(\frac{\mathrm{C}_{\mathrm{A}}}{3}-\frac{\mathrm{C}_{\mathrm{F}}}{2}+\frac{11}{12} \beta_{0}\right)}+\beta_{0}\left(\frac{55}{12}-4 \zeta_{3}\right)\right] \equiv \\
& 3 \mathrm{C}_{\mathrm{F}} \cdot d_{2}=3 \mathrm{C}_{\mathrm{F}} \cdot\left[\left(\frac{\mathrm{C}_{\mathrm{A}}}{3}-\frac{\mathrm{C}_{\mathrm{F}}}{2}\right)+\beta_{0}\left(\frac{11}{2}-4 \zeta_{3}\right)\right],
\end{aligned}
$$

and is consistent with the standard BLM result [1], which is given by eq. (3.10b). Indeed, the presentation of $D^{\mathrm{EM}}$ as the decomposition (2.10) has a rather "technical" sense, and for partial orders they can be presented in another form. For instance, in the expressions in the r.h.s. of eqs. (3.7) the anomalous dimension terms do not appear explicitly. It happens that in contrast to the initial polarization function $\Pi^{\mathrm{EM}}\left(L, a_{s}\right)$ that defines in eq. (2.11) the anomalous dimension $\gamma_{\mathrm{ph}}^{\mathrm{EM}}$, the RGI quantity $D^{\mathrm{EM}}\left(L, a_{s}\right)$ does not have its own anomalous dimension. Therefore, there is no physical reason to prefer only the second term of eq. (2.10) (or, the second term in eq. (3.10a)), considering them as a unique origin of the $a_{s}$ renormalization, as was done in, e.g. , $[12,13]$.

A similar expression for $d_{3}^{\mathrm{NS}}$, which follows from eq. (2.10), reads

$$
d_{3}^{\mathrm{NS}} \equiv 3 \mathrm{C}_{\mathrm{F}} \cdot d_{3}=\frac{3}{4}\left[\gamma_{3}+\beta_{1} \Pi_{2}^{\mathrm{R}}+\beta_{0} 2 \Pi_{3}^{\mathrm{R}}\right]=\frac{3}{4}\left[\gamma_{3}+\beta_{1} \Pi_{2}^{\mathrm{R}}+\beta_{0}\left(\beta_{0} 2 \Pi_{3 \beta}^{\mathrm{R}}+2 \Pi_{30}^{\mathrm{R}}\right)\right] .
$$

The contribution of $\Pi_{2}^{\mathrm{R}}$ and of the decomposed parts of the term $\Pi_{3}^{\mathrm{R}}=\beta_{0} \Pi_{3 \beta}^{\mathrm{R}}+\Pi_{30}^{\mathrm{R}}$ in eq. (3.11) can be extracted directly from the results for $\Pi_{i}^{\mathrm{R}}$ in $[17]^{2}$ and reads

$$
\begin{aligned}
& \Pi_{2}^{\mathrm{R}}=2 \mathrm{C}_{\mathrm{F}}\left(\frac{55}{12}-4 \zeta_{3}\right) ; \quad \Pi_{3 \beta}^{\mathrm{R}}=\mathrm{C}_{\mathrm{F}}\left(\frac{3701}{54}-\frac{152}{3} \zeta_{3}\right) \\
& \Pi_{30}^{\mathrm{R}}=2 \mathrm{C}_{\mathrm{F}}\left[\mathrm{C}_{\mathrm{F}}\left(-\frac{143}{9}-\frac{148}{3} \zeta_{3}+80 \zeta_{5}\right)+\mathrm{C}_{\mathrm{A}}\left(\frac{146}{13}-8 \zeta_{3}-\frac{40}{3} \zeta_{5}\right)\right] .
\end{aligned}
$$

Note that the coefficient $\gamma_{3}$ of the photon anomalous dimension function of eq. (3.9) has its own $\beta$-expansion, namely

$$
\gamma_{3}=\beta_{0}^{2} \gamma_{3}[2]+\beta_{1} \gamma_{3}[0,1]+\beta_{0} \gamma_{3}[1]+\gamma_{3}[0],
$$

which will be discussed in section 4 . Therefore, the $\{\beta\}$-expanded result for $d_{3}^{\mathrm{NS}}$ in eq. (3.11) (or $d_{3}$ in eq. (2.9c)) is composed from the $\{\beta\}$-expansion of both parts of eq. (2.10). To

\footnotetext{
${ }^{2}$ The analytical expressions for the QED contributions to $\Pi_{2}^{\mathrm{R}}, \Pi_{3}^{\mathrm{R}}$ were earlier presented in [23].
} 
complete the consideration, we present here the explicit expressions for the elements of the $\{\beta\}$-expansion of $\gamma_{3}$, defined in eq. (3.13), in the case when QCD is supplemented with the MSSM multiplet of gluino:

$$
\begin{aligned}
\gamma_{3}[2] & =-\mathrm{C}_{\mathrm{F}} \frac{77}{27}=\frac{4}{3} \cdot\left[d_{3}^{\mathrm{NS}}[2]-C_{\mathrm{F}}\left(\frac{3701}{36}-76 \zeta_{3}\right)\right], \\
\gamma_{3}[1] & =4 \mathrm{C}_{\mathrm{F}}\left[\mathrm{C}_{\mathrm{A}}\left(-\frac{1249}{108}+\frac{104}{3} \zeta_{3}\right)-\mathrm{C}_{\mathrm{F}}\left(\frac{19}{9}+\frac{8}{3} \zeta_{3}\right)\right], \\
\gamma_{3}[0,1] & =4 C_{\mathrm{F}}\left(\frac{23}{6}-4 \zeta_{3}\right), \\
\gamma_{3}[0] & =4 C_{\mathrm{F}}\left[\left(\frac{523}{36}-72 \zeta_{3}\right) \mathrm{C}_{\mathrm{A}}^{2}+\frac{71}{3} \mathrm{C}_{\mathrm{A}} \mathrm{C}_{\mathrm{F}}-\frac{23}{2} \mathrm{C}_{\mathrm{F}}^{2}\right]=\frac{4}{3} \cdot d_{3}^{\mathrm{NS}}[0] .
\end{aligned}
$$

The r.h.s. of eqs. (3.14) are derived from the result for $\gamma_{p h}\left(n_{f}, n_{\tilde{g}}\right)$, obtained in [20] in the same way as it was done for the elements of the $\{\beta\}$-expansion of the $d_{3}$ coefficient of the Adler function determined in $[2,5,6]$ (these elements and the required $\beta$-function coefficients are presented in the appendix).

The value in the first equation (3.14a) can be easily checked from the explicit result in [17] (see eq. (3.13) there). The last equation in (3.14d) has a general reason: only $\gamma_{n}[0]$-terms determine the expressions for $d_{n}^{\mathrm{NS}}[0]$. This feature can be clearly seen from the expression of eq. (2.10). It also clarifies that the $\beta$-dependent contributions to the elements defined in (2.9) are formed by the corresponding contributions from both $\gamma_{\mathrm{ph}}^{\mathrm{EM}}$ and $\Pi^{\mathrm{EM}}$. For example, substituting eqs. (3.14) into eq. (3.11) one can obtain the following structure of the $d_{3}^{\mathrm{NS}}$-term:

$$
d_{3}^{\mathrm{NS}}=\frac{3}{4}\left[\gamma_{3}[0]+\beta_{0}\left(\gamma_{3}[1]+2 \Pi_{30}^{\mathrm{R}}\right)+\beta_{1}\left(\gamma_{3}[0,1]+\Pi_{2}^{\mathrm{R}}\right)+\beta_{0}^{2}\left(\gamma_{3}[2]+2 \Pi_{3 \beta}^{\mathrm{R}}\right)\right],
$$

where

$$
d_{3}^{\mathrm{NS}}[2]=\frac{3}{4}\left(\gamma_{3}[2]+2 \Pi_{3 \beta}^{\mathrm{R}}\right), d_{3}^{\mathrm{NS}}[0,1]=\frac{3}{4}\left(\gamma_{3}[0,1]+\Pi_{2}^{\mathrm{R}}\right), d_{3}^{\mathrm{NS}}[1]=\frac{3}{4}\left(\gamma_{3}[1]+2 \Pi_{3,0}^{\mathrm{R}}\right) .
$$

In section 4, we shall discuss the origin of this decomposition using the language of the incomplete BPHZ $R$-operation, i.e., the $R^{\prime}$-operation.

\section{The structure of the $R^{\prime}$-operation and the $\{\beta\}$-expansion}

Our goal here is to trace the form of the expansion (2.9c) in regard to the term $\gamma_{3}$ of anomalous dimension $\gamma_{\mathrm{ph}}^{\mathrm{EM}}$ that, in turn, enters into $d_{3}$ by means of presentation (2.10), (3.15). The method we use is more general and relates the structure of the $\{\beta\}$-expansion to the structure of various contractions of the $R^{\prime}$-operation for any perturbative order. To be more precise these $R^{\prime}$-operation contractions of the subgraphs form the corresponding contributions to the $\beta_{i}$ coefficients of the $\beta$-function.

We start with the definition of the renormalization constant $Z$ in the MS-scheme [24],

$$
Z=1-\hat{K} R^{\prime}(G)
$$


where $G$ denotes the set of the corresponding diagrams (or a single one in the case of partial contribution) and $\hat{K}$ separates out poles in $\varepsilon$. One can rewrite expression (2.11) for the anomalous dimension $\gamma_{\mathrm{ph}}^{\mathrm{EM}}$ :

$$
\gamma_{\mathrm{ph}}^{\mathrm{EM}}=-\partial_{a_{s}}\left[a_{s} Z^{(1)}\right]=\partial_{a_{s}}\left[a_{s} \hat{K}_{1} R^{\prime}(G)\right]=\left.4 \hat{K}_{1} R^{\prime}(G)\right|_{4 \text {-loops }}
$$

where the last equality is written for the three-order contribution $a_{s}^{3} \gamma_{3}$. Here $R^{\prime}-$ the incomplete BPHZ $R$-operation, $R=R^{\prime}-\hat{K} R^{\prime}$ [14], i.e., the $R^{\prime}$-operation subtracts all the subdivergences of internal non-intersecting subgraphs but does not subtract an overall divergence $\hat{K} R^{\prime}$ of a diagram, see, e.g. , $[16,25] ; Z^{(1)}=-\hat{K}_{1} R^{\prime}(G)$ where $\hat{K}_{1}$ picks out the coefficient at the first pole. We will not present here the definition of the well-known $R^{\prime}$-operation, instead of that we will refer an interested reader to the detailed article [25] containing a number of appropriate examples and notations, which are used below.

One can classify the origins of different contributions to $\gamma_{3}$ in the expansion

$$
\gamma_{3}=\beta_{0}^{2} \gamma_{3}[2]+\beta_{1} \gamma_{3}[0,1]+\beta_{0} \gamma_{3}[1]+\gamma_{3}[0]
$$

and relate this $\beta$-expansion to the elements of the structure of the $\left(R^{\prime}-\mathbb{1}\right)$ operation in the last equality in the r.h.s. of eq. (4.2). In this notation the contribution to the $\beta$-function can be obtained as

$$
\beta\left(a_{s}\right)=a_{s}^{2} \partial_{a_{s}} Z_{g}^{(1)}=a_{s}^{2} \partial_{a_{s}}\left[-\hat{K}_{1} R^{\prime}\left(G^{g}\right)\right]=-a_{s} j\left[\hat{K}_{1} R^{\prime}\left(G^{g}\right)\right]
$$

where $Z_{g}$ is a contribution to the charge renormalization constant and $j$ is the power in $a_{s}$, which enter the subgraph $G^{g}$. Further, we shall classify different contributions to $\gamma_{3}$ following different kinds of contractions of subgraphs of the corresponding 4-loops diagrams $G_{4}$. Every contraction of the subgraph $G^{(l)}$ in $\hat{K}_{1} R^{\prime}\left(G_{4}\right)$ is related to either the renormalization of the charge $g\left(g^{2} /(4 \pi)=\alpha_{s}=4 \pi a_{s}\right)$ where $G^{g}$ is the subgraph that finally contributes to $\beta\left(a_{s}\right)$ through $Z_{g}^{(1)}$ in eq. (4.3), or the renormalization of electromagnetic vertex $J_{\mu}^{\mathrm{EM}}$ and of its legs, with the related $G^{J}$ subgraphs. The later renormalizations cancels in the sum due to the Ward identities (WI) [15].

Let us clarify this more formal proof considering all possible contraction of the subgraphs.

(i) Diagrams $G_{4}$ may admit only 2 contractions of the 1-loop intrinsic subgraphs $G_{1}^{(l)}$ under the $R^{\prime}$-operation. Let us choose those subgraphs, $G^{g}$, that renormalize the intrinsic charges $g$ thus form the renormalization constant $Z_{g}$, see the upper part in figure 1 . The residual of any diagrams under these contractions, $\Gamma^{\text {rest(1,1) }}$, reduces to sum of three diagrams shown in the lower part in figure 1.

This residual $\Gamma^{\mathrm{rest}(1,1)}=G_{4} \backslash G_{1}^{g(l)} \backslash G_{1}^{g(m)}$, resulting 3 after two one-loop contractions of $G_{4}$, is unique for any kind of contractions. The sum $\sum_{l}\left(\hat{K} G_{1}^{g(l)}\right) \sim \beta_{0}$ (here

\footnotetext{
${ }^{3}$ Sign " $\backslash$ "in an expression " $A \backslash B$ " means the subtractions of the subgraph $B$ from the graph $A$.
} 

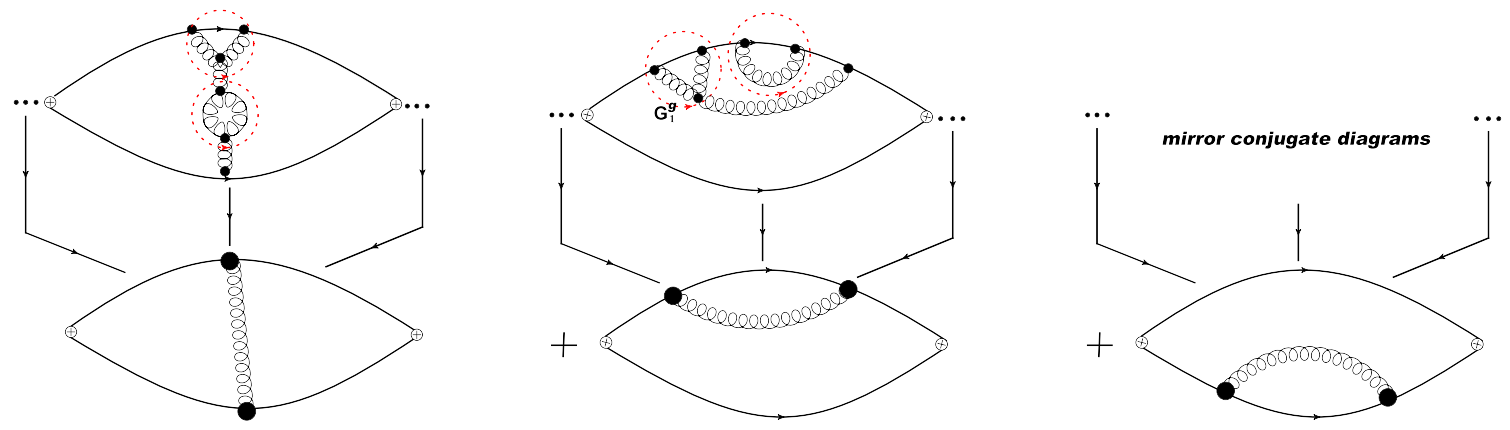

Figure 1. Upper row: the samples of 1-loop contractions, the dashed circle (in red) around graph $G$ denotes the operation $-\hat{K} R^{\prime}(G)$; here $\oplus$ is the electromagnetic vertex $J_{\mu}^{\text {EM }}$. Lower row: the diagrams, which determine the reminder $\Gamma^{\text {rest(1,1) }}$ after two of 1-loop $G_{1}$ contractions of the 4-loop diagrams $G_{4}$.

$\left.R^{\prime} G_{1}=G_{1}\right)$; therefore, the contribution to $\gamma_{3}$ after 2 contractions is

$$
\left[\hat{K}_{1} \sum_{m, l} \Gamma^{\mathrm{rest}(1,1)}(\varepsilon) \cdot\left(-\hat{K} G_{1}^{(l)}\right)\left(-\hat{K} G_{1}^{(m)}\right) \sim \Gamma_{\varepsilon}^{\mathrm{rest}(1,1)} \cdot \beta_{0}^{2}\right] \stackrel{\text { contr. }(1,1)}{\longrightarrow} \gamma_{3}[2] \cdot \beta_{0}^{2},
$$

where $\Gamma_{\varepsilon}^{\text {rest }(1,1)}=\left.\left(\Gamma^{\mathrm{rest}(1,1)}(\varepsilon)\right)^{\prime}\right|_{\varepsilon=0}$. After the sum over all contractions $(i, j)$ one obtains the factor $\beta_{0}^{2}$. The contributions of those one-loop contracted subgraphs that include $J^{\mathrm{EM}}$ vertices are cancelled by the contracted self-energy parts of fermions due to WI. The mixed cases including the contractions of the $G_{1}^{J}$ and $G^{g}$ subgraphs should result in zero by the same reason. Finally, the term $\Gamma_{\varepsilon}^{\text {rest(1,1) }}$ contributes to the corresponding $\gamma_{3}[2]$ element of the $\beta$-expansion.

(ii) The different contractions of the 2-loop subgraphs $G_{2}^{g(k)}$ that renormalize charges, lead to the $\beta_{1}$-part of the expansion at the single residual $\Gamma^{\mathrm{rest}(2)}=G_{4} \backslash G_{2}^{g(k)}$ of the diagram

$$
\left[\hat{K}_{1} \sum_{k} \Gamma^{\mathrm{rest}(2)}(\varepsilon)\left(-\hat{K} R^{\prime}\left(G_{2}^{(k)}\right)\right) \sim \Gamma_{0}^{\mathrm{rest}(2)} \beta_{1}+\Gamma_{\varepsilon}^{\mathrm{rest}(2)} \beta_{0}^{2}\right] \stackrel{\text { contr.(2) }}{\longrightarrow} \gamma_{3}[0,1] \cdot \beta_{1}+\ldots,
$$

where $\Gamma_{0}^{\mathrm{rest}(2)}=\Gamma^{\mathrm{rest}(1,1)}(0)$ is the part of $\Gamma^{\mathrm{rest}(2)}$ finite in $\varepsilon$. These terms contribute to the element $\gamma_{3}[0,1]$. Besides, the high pole $\left(1 / \varepsilon^{2}\right)$ of these contractions contribute to the term $\sim \Gamma_{\varepsilon}^{\text {rest }(2)} \cdot \beta_{0}^{2}$, i.e., again to $\gamma_{3}[2] \beta_{0}^{2}$. The contributions from the contracted $G_{2}^{J}$ subgraphs are factorized with the single residual given by the diagrams in the lower row of figure 1. Due to the WI the sum is zero, i.e. , $\sum_{l}\left(-\hat{K} R^{\prime}\left(G_{2}^{J(l)}\right)\right)=0$.

(iii) All the contractions of the 3-loop subgraphs $G_{3}^{(k)}$ of $G_{4}$ become the $G_{3}^{J(k)}$ subgraphs. One can verify that in this case the single residual $\Gamma^{\mathrm{rest}(3)}(\varepsilon)$ is given by the contribution of the simple quark loop $\Pi_{1}$, while the sum $\sum_{m}\left(-\hat{K} R^{\prime}\left(G_{3}^{J(m)}\right)\right)=0$ again due to the WI. 
(vi) The contractions of the 1-loop subgraphs $G_{1}^{g(k)}$ that form $Z_{g}$ of a certain charge $g$ contribute to the $\beta_{0}$-term and form in part the element $\gamma_{3}[1]$, namely

$$
\left[\hat{K}_{1} \sum_{k} \Gamma^{\mathrm{rest}(1 \mathrm{k})}(\varepsilon) \cdot\left(-\hat{K} R^{\prime}\left(G_{1}^{g(k)}\right)\right) \sim \Gamma_{0}^{\mathrm{rest}(1)} \cdot \beta_{0}+\ldots\right] \stackrel{\text { contr. }(1)}{\longrightarrow} \gamma_{3}[1] \cdot \beta_{0}+\ldots
$$

Here $\Gamma^{\text {rest(1)k }}=G_{4} \backslash G_{1}^{g(k)}$ are the same for a set of subgraphs, the common coefficient $\Gamma_{0}^{\text {rest(1) }}$ is a sum of the $\Gamma^{\text {rest(1)k }}$ terms. Of course these kinds of contributions can produce the $\beta_{0}^{2}$-term also, if $\Gamma_{0}^{\mathrm{rest}(1)}$ contains $\beta_{0}$. The latter possibility is indicated in (4.6) by dots.

The previous analysis is enough to fix the structure of the $\gamma_{3}$ coefficient, but not the values of $\gamma_{3}[\ldots]$ elements. We realize that the results in the items (i) - (vi) are not the single source of the $\beta$-terms in the expansion, the term $\hat{K}_{1}\left(G_{4}\right)$ formed from the subgraph $G_{4}$ without any contractions contributes too. Therefore

$$
\left[\hat{K}_{1}\left(G_{4}\right) \sim \beta_{0}^{2} \cdot(\ldots)+\beta_{1} \cdot(\ldots)+\beta_{0} \cdot(\ldots)+C\right] \stackrel{\text { contr. }(0)}{\longrightarrow} \gamma_{3}
$$

may contain any of the aforementioned elements of the $\beta$-structure and, in addition, the independent of $\beta_{0}$ and $\beta_{1}$ contribution, denoted by $C$. Finally, we can conclude that the structure of the $\{\beta\}$-expansion of $\gamma_{3}$ appears as a natural result of different contractions $\left(-\hat{K} R^{\prime}\left(G_{1,2, \ldots}^{g}\right)\right)$ of the subgraphs $G_{1,2, \ldots}^{g}$ under the action of $R^{\prime}$-operation.

\section{Generalized $\{\beta, \gamma\}$-expansion}

Another kind of the $\{\beta\}$-expansion appears for RG covariant (RGC) quantities, those that have their own anomalous dimension For these RG covariant dimensionless one-scales quantities, say Green function $S\left(Q^{2} / \mu^{2} ; a_{s}\left(\mu^{2}\right)\right)$, the well-known RG transform is

$$
\begin{aligned}
S\left(Q^{2} / \mu^{2} ; a_{s}\left(\mu^{2}\right)\right) & =\hat{z}\left(t, a_{s}\left(\mu^{2}\right)\right) \cdot S\left(Q^{2} / \mu^{2} \frac{1}{t} ; \bar{a}_{s}\left(t, a_{s}\left(\mu^{2}\right)\right)\right) \\
\text { where } \hat{z}\left(t, a_{s}\left(\mu^{2}\right)\right) & =\exp \left\{\int_{1}^{t} \gamma_{S}\left(\bar{a}_{s}\left(\tau, a_{s}\left(\mu^{2}\right)\right) \frac{d \tau}{\tau}\right\}, \bar{a}_{s}\left(1, a_{s}\left(\mu^{2}\right)\right)=a_{s}\left(\mu^{2}\right),\right.
\end{aligned}
$$

for the simplest case without mixing, ${ }^{4}$ where the scale extension factor $t=\mu^{\prime 2} / \mu^{2}$ and the anomalous dimensions of $S$ is $\gamma_{S}\left(a_{s}\right)=\sum_{j=1} a_{s}^{j} \gamma_{j}$. Given $\gamma_{S}\left(a_{s}\right)=0$ one returns to the condition (2.7) for RGI quantities in section 2. Let us consider the perturbative expansion of $S\left(1 ; \bar{a}_{s}\left(Q^{2}\right)\right)$ at the value $\mu^{2}=Q^{2}, s_{0}=1$,

$$
S\left(1 ; \bar{a}_{s}\right)=1+\sum_{n=1} \bar{a}_{s}^{n} s_{n}
$$

and let analyse the structure of the expansion coefficients $s_{n}$ in comparison with the RGI case in eq. (2.9). The decomposition of $s_{n}$ should include along with elements of the

\footnotetext{
${ }^{4}$ For the case of mixing the $\hat{z}$ and $\gamma_{S}$ are matrixes.
} 
$\{\beta\}$-expansion also the elements with $\gamma_{j}$ that we will separate from the first ones. The difference with the standard $\{\beta\}$-expansion reveals itself starting with $s_{1}$, which includes now the contribution with $\gamma_{0}$ and are marked in (5.3) by the bold font, while the standard $\beta$-terms - by the italic one, namely

$$
\begin{aligned}
s_{1}=s_{1}[0] & +\gamma_{0} \boldsymbol{s}_{\mathbf{1}}[\mathbf{0} \mid \mathbf{1}] \\
s_{2}=s_{2}[0] & +\beta_{0} s_{2}[1 \mid 0]+ \\
& +\gamma_{1} \boldsymbol{s}_{\mathbf{2}}[\mathbf{0} \mid \mathbf{0}, \mathbf{1}]+\gamma_{0}^{2} \frac{1}{2} \boldsymbol{s}_{\mathbf{2}}[\mathbf{0} \mid \mathbf{2}]-\gamma_{0} \beta_{0} \frac{1}{2} s_{\mathbf{2}}[\mathbf{1} \mid \mathbf{1}] \\
s_{3}=s_{3}[0] & +\beta_{0}^{2} s_{3}[2 \mid 0]+\beta_{1} s_{3}[0,1 \mid 0]+\beta_{0} s_{3}[1 \mid 0]+ \\
& +\gamma_{2} \boldsymbol{s}_{\mathbf{3}}[\mathbf{0} \mid \mathbf{0}, \mathbf{0}, \mathbf{1}]-\frac{\gamma_{0}}{2} \beta_{1} \boldsymbol{s}_{\mathbf{3}}[\mathbf{0}, \mathbf{1} \mid \mathbf{1}]-\gamma_{1} \beta_{0} \boldsymbol{s}_{\mathbf{3}}[\mathbf{1} \mid \mathbf{0}, \mathbf{1}]-\gamma_{1} \gamma_{0} \boldsymbol{s}_{\mathbf{3}}[\mathbf{0} \mid \mathbf{1}, \mathbf{1}]+ \\
& +\frac{\gamma_{0}}{3} \beta_{0}^{2} s_{\mathbf{3}}[\mathbf{2} \mid \mathbf{1}]-\frac{\gamma_{0}^{2}}{2} \beta_{0} \boldsymbol{s}_{\mathbf{3}}[\mathbf{1} \mid \mathbf{2}]+\frac{\gamma_{0}^{3}}{3} \boldsymbol{s}_{\mathbf{3}}[\mathbf{0} \mid \mathbf{3}] \\
&
\end{aligned}
$$

The first two elements in the expansion of $s_{2}$ repeat the $\{\beta\}$-expansion, while the other new admissible elements are proportional to $\gamma_{0}$ or $\gamma_{1}$. The coefficients $(1 / 2)$ and sign are chosen in $(5.3 \mathrm{~b})$ in accordance with the perturbation expansion of the factor $\hat{z}\left(t, a_{s}\right)$ in eq. (5.4a),

$$
\begin{aligned}
\hat{z}\left(t, a_{s}\right)= & 1+a_{s} \gamma_{0} l+a_{s}^{2}\left(\gamma_{1}+\gamma_{0}^{2} \frac{1}{2} l-\gamma_{0} \beta_{0} \frac{1}{2} l\right) l+ \\
& +a_{s}^{3}\left[\gamma_{2}-\left(\frac{\gamma_{0}}{2} \beta_{1}+\gamma_{1}\left(\beta_{0}-\gamma_{0}\right)\right) l+\frac{\gamma_{0}}{3}\left(\beta_{0}-\frac{\gamma_{0}}{2}\right)\left(\beta_{0}-\gamma_{0}\right) l^{2}\right] l \\
& +O\left(a_{s}^{4}\right) ; \quad l=\ln (t) .
\end{aligned}
$$

The similar notations for the decomposition of $s_{3}$ in eq. (5.3c) follow to the expansion in (5.4b). All the admissible elements of the RG generators $\left(\beta, \gamma_{S}\right)$ for quantity $S$ should be taken into account in the decomposition of the coefficients $s_{n}$ (some of these elements may be equal to zero) as the "traces" of the RG factor $\hat{z}$. The notation we use in eq. (5.3) follows the rule: the first series $\{\beta\}$ before the separator in the square brackets of $s_{n}[\{\beta\} \mid\{\gamma\}]$ means the number of powers of $\beta_{i}$ in the $i$-position in full correspondence with the notation in eq. (2.9); see, e.g. , $\beta_{0} s_{2}[1 \mid 0]$ in (5.3b), or $\beta_{1} s_{3}[0,1 \mid 0]$ in (5.3c). The second series $\{\gamma\}$ stays after this separator, it counts the powers of $\gamma_{j}$ that accompanying this contribution, see, e.g. , $\gamma_{0} \boldsymbol{s}_{\mathbf{1}}[\mathbf{0} \mid \mathbf{1}]$. These new terms are presented in the second lines of $(5.3 \mathrm{~b}),(5.3 \mathrm{c})$ and are constructed just in the same manner as the ones for $\beta_{i}$. So we separate here the $\{\beta\}$-expansion from the $\{\gamma\}$ one.

The question is how we can use this new detailed decomposition of the series coefficients? For the purpose of the series optimization one can collect the terms with anomalous dimensions $\gamma_{i}$ in decompositions in eqs. (5.3) and transfer them into the common $\hat{z}$ factor starting with, e.g., the first order term $\gamma_{0} \boldsymbol{s}_{\mathbf{1}}[\mathbf{0} \mid \mathbf{1}]$ in (5.3a). This fixes the logarithmic shift $\Delta[5], \Delta=s_{1}[\mathbf{0} \mid \mathbf{1}]=\ln \left(Q^{2} / \mu^{2}\right)$, to the new normalization scale $Q^{2}$. The term $\beta_{0} \Delta$ corresponded this new scale should be removed from the first line in eq. (5.3b) in the second order and so on following to BLM procedure. We shall elaborate this kind of series optimization and apply them to certain quantities somewhere else. The main goal of this demonstration 
is to reveal the difference between the RGI and RGC quantities. In the first case, one has the $\{\beta\}$-expansion only, while for the last case, one should distinguish the $\beta$ and $\gamma$-terms in the decomposition of the expansion coefficients to form the common factor $\hat{z}$.

It should be mentioned that an optimization approach based on the $\{\beta, \gamma\}$-expansion should replace the PMC-type optimization approach for the concrete case of the optimization of the total decay width of the $H^{0} \rightarrow b \bar{b}$ decay process, considered in [26]. The importance of this consideration was emphasized in [27].

\section{Conclusion}

We discussed the $\{\beta\}$-expansion for renormalization group (RG) invariant quantities. The origins of this expansion are traced to the structure of the result of the incomplete BPHZ $R$-operation - the $R^{\prime}$-operation, considering the role of various contractions there. All the coupling renormalizations following from these contractions should be taken into account for the $\{\beta\}$-expansion. We illustrate our theoretical conclusion in the $O\left(a_{s}^{3}\right)$ order by means of analysis of the calculation scheme, when the nonsinglet Adler function $D^{\mathrm{NS}}$ is expressed through the photon anomalous dimension $\gamma_{P h}^{E M}\left(a_{s}\right)$. Note here that in [28] another special QCD approach was used to determine the coefficients of the $\{\beta\}$-expansion in eqs. $(2.9 \mathrm{c}),(2.9 \mathrm{~d})$. The detailed comparison of the results of its applications with the results obtained here is on the agenda.

We proposed a generalization of the $\{\beta\}$-expansion for the renormalization group covariant (RGC) quantities - the $\{\beta, \gamma\}$-expansion. This expansion can be the basis of a new optimization procedure for the RGC quantities.

\section{Acknowledgments}

We would like to thank S. J. Brodsky and M. Y. Kalmykov for the fruitful discussion.

The work of A.K. was supported by the Russian Science Foundation grant No. 14-2200161. The work of M.S. was supported in part by the BelRFFR-JINR, grant F16D-004 and by the Russian Foundation for Basic Research, Grant No. 14-01-00647.

\section{A Explicit formulas for the elements of $D$ and $\beta$-function coefficients}

For the Adler function $D^{\mathrm{NS}}$ obtained within QCD with light gluinos $n_{\tilde{g}}$ the elements of the $\{\beta\}$-expansion read

$$
\begin{aligned}
& d_{1}^{\mathrm{NS}}=3 \mathrm{C}_{\mathrm{F}} ; d_{1}=1 ; \\
& d_{2}[1]=\frac{11}{2}-4 \zeta_{3} ; \quad d_{2}[0]=\frac{\mathrm{C}_{\mathrm{A}}}{3}-\frac{\mathrm{C}_{\mathrm{F}}}{2} \\
& d_{3}[2]=\frac{302}{9}-\frac{76}{3} \zeta_{3} ; d_{3}[0,1]=\frac{101}{12}-8 \zeta_{3} ; \\
& d_{3}[1]=\mathrm{C}_{\mathrm{A}}\left(-\frac{3}{4}+\frac{80}{3} \zeta_{3}-\frac{40}{3} \zeta_{5}\right)-\mathrm{C}_{\mathrm{F}}\left(18+52 \zeta_{3}-80 \zeta_{5}\right) ; \\
& d_{3}[0]=\left(\frac{523}{36}-72 \zeta_{3}\right) \mathrm{C}_{\mathrm{A}}^{2}+\frac{71}{3} \mathrm{C}_{\mathrm{A}} \mathrm{C}_{\mathrm{F}}-\frac{23}{2} \mathrm{C}_{\mathrm{F}}^{2}
\end{aligned}
$$


The required $\beta$-function coefficients within the same scheme with the light gluinos $n_{\tilde{g}}$ [29] and the number $n_{f}$ of the quark flavors calculated in the $\overline{\mathrm{MS}}$ scheme are

$$
\begin{aligned}
\beta_{0}\left(n_{f}, n_{\tilde{g}}\right)= & \frac{11}{3} C_{A}-\frac{4}{3}\left(T_{R} n_{f}+\frac{n_{\tilde{g}} C_{A}}{2}\right) ; \\
\beta_{1}\left(n_{f}, n_{\tilde{g}}\right)= & \frac{34}{3} C_{A}^{2}-\frac{20}{3} C_{A}\left(T_{R} n_{f}+\frac{n_{\tilde{g}} C_{A}}{2}\right)-4\left(T_{R} n_{f} C_{\mathrm{F}}+\frac{n_{\tilde{g}} C_{A}}{2} C_{A}\right) ; \quad \text { (A.2a) } \\
\beta_{2}\left(n_{f}, n_{\tilde{g}}\right)= & \frac{2857}{54} C_{A}^{3}-n_{f} T_{R}\left(\frac{1415}{27} C_{A}^{2}+\frac{205}{9} C_{A} C_{F}-2 C_{F}^{2}\right)+\left(n_{f} T_{R}\right)^{2}\left(\frac{44}{9} C_{F}+\frac{158}{27} C_{A}\right) \\
& -\frac{988}{27} n_{\tilde{g}} C_{A}\left(C_{A}^{2}\right)+n_{\tilde{g}} C_{A} n_{f} T_{R}\left(\frac{22}{9} C_{A} C_{F}+\frac{224}{27} C_{A}^{2}\right)+\left(n_{\tilde{g}} C_{A}\right)^{2} \frac{145}{54} C_{A} \cdot \text { (A.2c) }
\end{aligned}
$$

The $\mathrm{N}^{3} \mathrm{LO}$ coefficient $\beta_{3}\left(n_{f}, n_{\tilde{g}}\right)$ has been obtained recently in the papers [30, 31].

For the sake of readers we present here also the $\gamma_{0,1,2}$ values from [17], rescaling them to our normalization of the coupling constant $a_{s}=\alpha_{s} /(4 \pi)$,

$$
\gamma_{0}=\frac{4}{3}, \gamma_{1}=4 \mathrm{C}_{\mathrm{F}}, \gamma_{2}=4 \mathrm{C}_{\mathrm{F}}\left(\frac{\mathrm{C}_{\mathrm{A}}}{3}-\frac{\mathrm{C}_{\mathrm{F}}}{2}+\frac{11}{12} \beta_{0}\right) .
$$

Open Access. This article is distributed under the terms of the Creative Commons Attribution License (CC-BY 4.0), which permits any use, distribution and reproduction in any medium, provided the original author(s) and source are credited.

\section{References}

[1] S.J. Brodsky, G.P. Lepage and P.B. Mackenzie, On the elimination of scale ambiguities in perturbative quantum chromodynamics, Phys. Rev. D 28 (1983) 228 [InSPIRE].

[2] S.V. Mikhailov, Generalization of BLM procedure and its scales in any order of $p Q C D:$ a practical approach, JHEP 06 (2007) 009 [hep-ph/0411397] [INSPIRE].

[3] X.-G. Wu, S.J. Brodsky and M. Mojaza, The renormalization scale-setting problem in QCD, Prog. Part. Nucl. Phys. 72 (2013) 44 [arXiv: 1302.0599] [InSPIRE].

[4] T. Gehrmann, N. Häfliger and P.F. Monni, BLM scale fixing in event shape distributions, Eur. Phys. J. C 74 (2014) 2896 [arXiv:1401.6809] [InSPIRE].

[5] A.L. Kataev and S.V. Mikhailov, Generalization of the Brodsky-Lepage-Mackenzie optimization within the $\{\beta\}$-expansion and the principle of maximal conformality, Phys. Rev. D 91 (2015) 014007 [arXiv: 1408.0122] [inSPIRE].

[6] A.L. Kataev and S.V. Mikhailov, New perturbation theory representation of the conformal symmetry breaking effects in gauge quantum field theory models, Theor. Math. Phys. 170 (2012) 139 [arXiv:1011.5248] [INSPIRE].

[7] A.L. Kataev and S.V. Mikhailov, $\{\beta\}$-expansion in $Q C D$, its conformal symmetry limit: theory + applications, Nucl. Part. Phys. Proc. 258-259 (2015) 45 [arXiv:1410.0554] [INSPIRE].

[8] A.L. Kataev, The generalized BLM approach to fix scale-dependence in QCD: the current status of investigations, J. Phys. Conf. Ser. 608 (2015) 012078 [arXiv:1411.2257] [INSPIRE]. 
[9] A.P. Bakulev, S.V. Mikhailov and N.G. Stefanis, Higher-order QCD perturbation theory in different schemes: from FOPT to CIPT to FAPT, JHEP 06 (2010) 085 [arXiv: 1004.4125] [INSPIRE].

[10] S.J. Brodsky and X.-G. Wu, Scale setting using the extended renormalization group and the principle of maximum conformality: the QCD coupling constant at four loops,

Phys. Rev. D 85 (2012) 034038 [Erratum ibid. D 86 (2012) 079903] [arXiv:1111.6175] [INSPIRE].

[11] M. Mojaza, S.J. Brodsky and X.-G. Wu, Systematic all-orders method to eliminate renormalization-scale and scheme ambiguities in perturbative $Q C D$, Phys. Rev. Lett. 110 (2013) 192001 [arXiv:1212.0049] [INSPIRE].

[12] S.J. Brodsky, M. Mojaza and X.-G. Wu, Systematic scale-setting to all orders: the principle of maximum conformality and commensurate scale relations, Phys. Rev. D 89 (2014) 014027 [arXiv: 1304.4631] [INSPIRE].

[13] H.-H. Ma, X.-G. Wu, Y. Ma, S.J. Brodsky and M. Mojaza, Setting the renormalization scale in perturbative $Q C D$ : comparisons of the principle of maximum conformality with the sequential extended Brodsky-Lepage-Mackenzie approach, Phys. Rev. D 91 (2015) 094028 [arXiv: 1504.01260] [INSPIRE].

[14] N.N. Bogoliubov and D.V. Shirkov, Introduction to the theory of quantized fields, IV edition, in Collection of scientific works in 12 volumes, N.N. Bogoliubov, volume 10, section 30, Nauka, Moscow Russia (2008) [INSPIRE].

[15] K.G. Chetyrkin, A.L. Kataev and F.V. Tkachov, Computation of the $\alpha_{s}^{2}$ correction $\sigma_{\text {tot }}$ $\left(e^{+} e^{-} \rightarrow\right.$ hadrons) in $Q C D$, preprint IYaI-P-0170, (1980) [INSPIRE].

[16] K.G. Chetyrkin, A.L. Kataev and F.V. Tkachov, New approach to evaluation of multiloop Feynman integrals: the Gegenbauer polynomial x space technique, Nucl. Phys. B 174 (1980) 345 [INSPIRE].

[17] P.A. Baikov, K.G. Chetyrkin, J.H. Kuhn and J. Rittinger, Vector correlator in massless $Q C D$ at order $O\left(\alpha_{s}^{4}\right)$ and the QED $\beta$-function at five loop, JHEP 07 (2012) 017 [arXiv: 1206.1284] [INSPIRE].

[18] S.G. Gorishnii, A.L. Kataev and S.A. Larin, The $O\left(\alpha_{s}^{3}\right)$-corrections to $\sigma_{\text {tot }}\left(e^{+} e^{-} \rightarrow\right.$ hadrons $)$ and $\Gamma\left(\tau^{-} \rightarrow \nu_{\tau}+\right.$ hadrons) in QCD, Phys. Lett. B 259 (1991) 144 [INSPIRE].

[19] L.R. Surguladze and M.A. Samuel, Total hadronic cross-section in $e^{+} e^{-}$annihilation at the four loop level of perturbative QCD, Phys. Rev. Lett. 66 (1991) 560 [Erratum ibid. 66 (1991) 2416] [INSPIRE].

[20] K.G. Chetyrkin, Corrections of order $\alpha_{s}^{3}$ to $R_{\text {had }}$ in $p Q C D$ with light gluinos, Phys. Lett. B 391 (1997) 402 [hep-ph/9608480] [INSPIRE].

[21] S.L. Adler, Some simple vacuum polarization phenomenology: $e^{+} e^{-} \rightarrow$ hadrons: the $\mu$-mesic atom X-ray discrepancy and $(g-2)$ of the muon, Phys. Rev. D 10 (1974) 3714 [inSPIRE].

[22] K.G. Chetyrkin, A.L. Kataev and F.V. Tkachov, Higher order corrections to $\sigma_{\text {tot }}\left(e^{+} e^{-} \rightarrow\right.$ hadrons) in quantum chromodynamics, Phys. Lett. B 85 (1979) 277 [INSPIRE].

[23] S.G. Gorishnii, A.L. Kataev and S.A. Larin, The three loop QED contributions to the photon vacuum polarization function in the MS scheme and the four loop corrections to the QED B-function in the on-shell scheme, Phys. Lett. B 273 (1991) 141 [Erratum ibid. B 275 (1992) 512] [Erratum ibid. B 341 (1995) 448] [INSPIRE]. 
[24] A.A. Vladimirov, Methods of multiloop calculations and the renormalization group analysis of $\phi^{4}$ theory, Theor. Math. Phys. 36 (1979) 732 [Teor. Mat. Fiz. 36 (1978) 271] [InSPIRE].

[25] O.V. Tarasov and A.A. Vladimirov, Three loop calculations in non-Abelian gauge theories, preprint JINR-E2-80-483, (1980) [Phys. Part. Nucl. 44 (2013) 791] [arXiv:1301.5645] [INSPIRE].

[26] S.-Q. Wang, X.-G. Wu, X.-C. Zheng, J.-M. Shen and Q.-L. Zhang, The Higgs boson inclusive decay channels $H \rightarrow b \bar{b}$ and $H \rightarrow g g$ up to four-loop level, Eur. Phys. J. C 74 (2014) 2825 [arXiv: 1308.6364] [INSPIRE].

[27] A.A. Petrov, S. Pokorski, J.D. Wells and Z. Zhang, Role of low-energy observables in precision Higgs boson analyses, Phys. Rev. D 91 (2015) 073001 [arXiv: 1501.02803] [INSPIRE].

[28] G. Cvetič and A.L. Kataev, Adler function and Bjorken polarized sum rule: perturbation expansions in powers of the $\mathrm{SU}\left(N_{c}\right)$ conformal anomaly and studies of the conformal symmetry limit, Phys. Rev. D 94 (2016) 014006 [arXiv:1604.00509] [INSPIRE].

[29] L. Clavelli, P.W. Coulter and L.R. Surguladze, Gluino contribution to the three loop $\beta$-function in the minimal supersymmetric Standard Model, Phys. Rev. D 55 (1997) 4268 [hep-ph/9611355] [INSPIRE].

[30] M.F. Zoller, Four-loop QCD $\beta$-function with different fermion representations of the gauge group, JHEP 10 (2016) 118 [arXiv: 1608.08982] [INSPIRE].

[31] A.V. Bednyakov and A.F. Pikelner, On the four-loop strong coupling $\beta$-function in the SM, EPJ Web Conf. 125 (2016) 04008 [arXiv: 1609.02597] [INSPIRE]. 